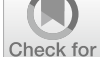

Check for

updates

Cite as

Nano-Micro Lett.

(2021) 13:148

Received: 16 March 2021

Accepted: 11 May 2021

Published online: 22 June 2021

(C) The Author(s) 2021

\section{Direct Ink Writing of Highly Conductive MXene Frames for Tunable Electromagnetic Interference Shielding and Electromagnetic Wave-Induced Thermochromism}

\author{
Xinyu $\mathrm{Wu}^{1,2}$, Tingxiang $\mathrm{Tu}^{1}$, Yang Dai ${ }^{1}$, Pingping Tang ${ }^{1}$, Yu Zhang ${ }^{1}$, Zhiming Deng ${ }^{2}$,
} Lulu $\mathrm{Li}^{2}$, Hao-Bin Zhang ${ }^{1,2} \bowtie$, Zhong-Zhen $\mathrm{Yu}^{2,3} \bowtie$

\title{
HIGHLIGHTS
}

- 3D printing of MXene frames with tunable electromagnetic interference shielding efficiency is demonstrated.

- Highly conductive MXene frames are reinforced by cross-linking with aluminum ions.

- Electromagnetic wave is visualized by electromagnetic-thermochromic MXene patterns.

\begin{abstract}
The highly integrated and miniaturized next-generation electronic products call for high-performance electromagnetic interference (EMI) shielding materials to assure the normal operation of their closely assembled components. However, the most current techniques are not adequate for the fabrication of shielding materials with programmable structure and controllable shielding efficiency. Herein, we demonstrate the direct ink writing of robust and highly conductive $\mathrm{Ti}_{3} \mathrm{C}_{2} \mathrm{~T}_{\mathrm{x}}$ MXene frames with customizable structures by using $\mathrm{MXene} / \mathrm{AlOOH}$ inks for tunable EMI shielding and electromagnetic wave-induced thermochromism applications. The as-printed frames are reinforced by immersing in $\mathrm{AlCl}_{3} / \mathrm{HCl}$ solution to remove the electrically insulating $\mathrm{AlOOH}$ nanoparticles, as well as cross-link the MXene sheets and fuse the filament interfaces with aluminum ions. After freeze-drying, the resultant robust and porous MXene frames exhibit tunable EMI shielding efficiencies in the range of 25-80 dB

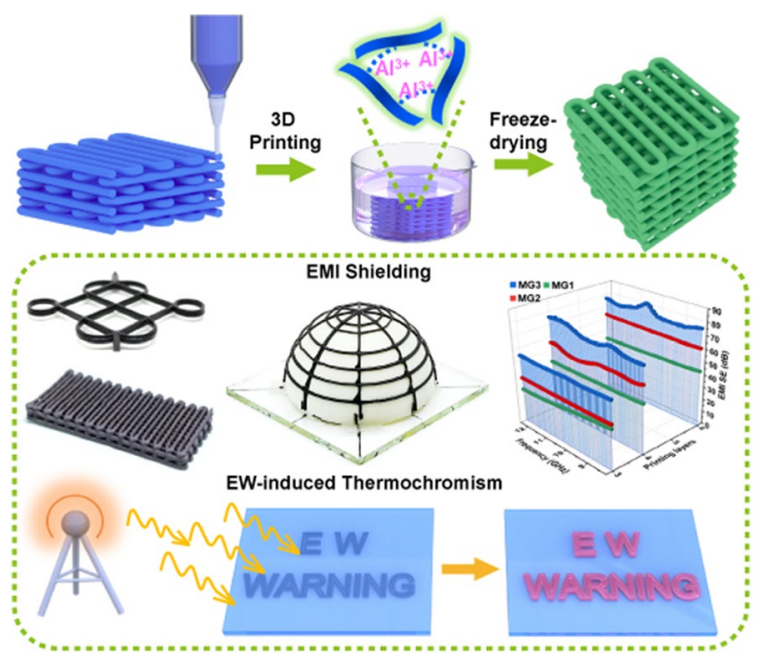
with the highest electrical conductivity of $5323 \mathrm{~S} \mathrm{~m}^{-1}$. Furthermore, an electromagnetic wave-induced thermochromic MXene pattern is assembled by coating and curing with thermochromic polydimethylsiloxane on a printed MXene pattern, and its color can be changed from blue to red under the high-intensity electromagnetic irradiation. This work demonstrates a direct ink printing of customizable EMI frames and patterns for tuning EMI shielding efficiency and visualizing electromagnetic waves.
\end{abstract}

KEYWORDS MXene; Electromagnetic interference shielding; Direct ink writing; Electrical conductivity; Thermochromism

Hao-Bin Zhang, zhanghaobin@mail.buct.edu.cn; Zhong-Zhen Yu, yuzz@mail.buct.edu.cn

1 State Key Laboratory of Organic-Inorganic Composites, College of Materials Science and Engineering, Beijing University of Chemical Technology, Beijing 100029, People's Republic of China

2 Beijing Key Laboratory of Advanced Functional Polymer Composites, Beijing University of Chemical Technology, Beijing 100029, People's Republic of China

3 Beijing Advanced Innovation Center for Soft Matter Science and Engineering, Beijing University of Chemical Technology, Beijing 100029, People's Republic of China 


\section{Introduction}

Electromagnetic waves can propagate in the free space without any physical media, making it possible for cross-regional information communication. Although electromagnetic waves cannot be recognized with naked eyes, their existence relies on alternating electromagnetic fields, carrying a lot of electromagnetic energy to achieve long-distance information transmissions. To mitigate radiation and interference of electromagnetic waves to the human body or sophisticated electronic equipment, the electromagnetic energy should be reflected, absorbed, or dissipated to heat by electromagnetic radiation shielding materials [1].

Two-dimensional (2D) $\mathrm{Ti}_{3} \mathrm{C}_{2} \mathrm{~T}_{\mathrm{x}}$ MXene is firstly applied for electromagnetic interference (EMI) shielding in 2016 on the basis of its outstanding electrical conductivity, abundant surface hydrophilic functional groups, large specific surface area, and ease of process [2]. Till now, various MXene films $[3,4]$, wearable fabrics $[5,6]$, as well as multifunctional composites [7, 8] are developed with excellent EMI shielding performances. Three-dimensional (3D) EMI shielding materials are usually designed with layer-by-layer, porous, or isolated internal structures to attenuate electromagnetic waves effectively by their conductive or magnetic components $[9,10]$. Porous structures can be constructed in the forms of aerogels or foams by random, directional [11, 12] and bidirectional freeze-drying [13, 14], dip-coating with foam templates [15], heterogeneous interface assembly $[16,17]$, and so on. Although the 3D MXene architectures prepared by the above approaches exhibit excellent EMI shielding performances, their shapes and internal structures are restricted by the forming molds and/or templates. It is currently difficult to prepare 3D MXene architectures with customized configurations.

In EMI shielding scenarios, conventional metal shields are gradually replaced by conformal shielding materials and compartmental shielding materials. To meet the requirement of shape-conformability, direct ink writing (DIW) is increasingly used as a cost-effective and easy-forming 3D printing technology for producing devices and equipment [18]. Its mild processing temperature and the advantage of integrating multiple materials make it a favorable choice for 3D composites [19, 20]. Recently, MXene-based architectures are fabricated on the basis of the DIW. Zhang et al. [21] designed complex patterns by using an additive-free aqueous ink of MXene with a concentration of $\sim 36 \mathrm{mg} \mathrm{mL}^{-1}$. Yang et al. [22] printed MXene microlattice and rectangular hollow prism with large $\mathrm{Ti}_{3} \mathrm{C}_{2} \mathrm{~T}_{\mathrm{x}}$ flakes in a large concentration range of $15-50 \mathrm{mg} \mathrm{mL}^{-1}$. Orangi et al. [23] used superabsorbent polymer beads to absorb water in the $\mathrm{Ti}_{3} \mathrm{C}_{2} \mathrm{~T}_{\mathrm{x}}$ suspension and printed filaments with a predetermined morphology at the $\mathrm{Ti}_{3} \mathrm{C}_{2} \mathrm{~T}_{\mathrm{x}}$ concentration of $28.9 \mathrm{wt} \%$. The printable MXene inks are also obtained by synergizing with different additives. For example, graphene oxide sheets and carbon nanotubes (CNTs) are incorporated with nitrogendoped MXene $\left(\mathrm{N}-\mathrm{Ti}_{3} \mathrm{C}_{2} \mathrm{~T}_{\mathrm{x}}\right)$ for the fabrication of sodium-ion mixture capacitor electrodes [24]. Silver nanowires, $\mathrm{MnO}_{2}$ nanowires, and fullerenes are used as MXene ink additives for constructing stretchable supercapacitors [25]. To the best of our knowledge, there is still rare report on direct ink writing of conformable MXene-based EMI shielding materials.

As an extrusion printing technology, DIW facilitates the formation of a continuous conductance path inside an entire 3D architecture and meets the prerequisite of high electrical conductivity for EMI shielding [26, 27]. The flexibility of design makes it possible to prepare periodic unit structures on the basis of electromagnetic theories [28, 29]. Wang et al. [30] prepared 3D core-shell structured liquid metal/elastomer composites with adjustable shielding efficiency by varying printing layers. Other architectures, like split-ring resonator and wire strip with a negative index of refraction [31], Miura-Ori origami structure with the frequency-selective surface [32], and saw-tooth folding films with tunable electromagnetic shielding [33], can be constructed with the DIW technology. This technology is effective in bridging the gap between the conductive MXene sheets and the customized electromagnetic shielding devices.

Herein, we demonstrate a DIW route to construct highly conductive MXene-based frames with customizable architecture for EMI shielding and electromagnetic-thermochromism applications. The flowability of the MXene ink is coordinated by adding $\mathrm{AlOOH}$ nanoparticles to ensure its printable ability and the structural stability of printed architectures. The printed MXene/AlOOH (MA) filaments are stacked up layer by layer and closely overlapped with each other, constructing the internally connected 3D conductive frame. After freeze-drying, the electrically insulating $\mathrm{AlOOH}$ nanoparticles are removed by etching with hydrochloric acid to generate pores inside the printed architecture, while the aluminum ions cross-link the MXene sheets to 
enhance the structural stability of the printed architecture, enabling it to sustain 3000 times load of its own weight. The gelled MXene frame (MG) exhibits a high electrical conductivity of 5,324 S m$~_{-1}$, and its EMI shielding effectiveness is over $80 \mathrm{~dB}$ in the $\mathrm{X}$-band. Furthermore, the printed MG patterns are coated and cured with a synthesized thermochromic polymer to realize the visualization of electromagnetic waves by naked eyes on the basis of the electromagnetic energy and thermal energy conversion principle.

\section{Experimental}

\subsection{Materials}

$\mathrm{Ti}_{3} \mathrm{AlC}_{2}$ powder (400 mesh) and $\mathrm{LiF}$ (purity $99.99 \%$ ) were purchased from Jilin 11 Technology (China) and Aladdin (China), respectively. AlOOH nanoparticles with an average particle size of $50 \mathrm{~nm}$ were provided by Beijing Dekedaojin (China). $\mathrm{AlCl}_{3} \cdot 6 \mathrm{H}_{2} \mathrm{O}$ was supplied by Xilong Scientific Company. Polydimethylsiloxane (SYLGARD 184, PDMS) prepolymer and its curing agent were purchased from Dow Corning (USA) and used with a mass ratio of 10:1. Hydrochloric acid ( $\mathrm{HCl}, 37 \mathrm{wt} \%)$, absolute ethanol, and chloroform $\left(\mathrm{CHCl}_{3}\right)$ were all provided by Beijing Chemical Reagents (China). 10,12-pentacosadiynoicacid (PCDA, purity 98\%) was purchased from Alfa Aesar Chemicals. PCDA was dissolved in absolute ethanol and filtrated to remove its polymerized component.

\subsection{Preparation of Few-Layer $\mathrm{Ti}_{3} \mathrm{C}_{2} \mathrm{~T}_{\mathrm{x}}$ MXene Sheets by Etching of $\mathrm{Ti}_{3} \mathrm{AlC}_{2}$ Flakes}

$\mathrm{LiF}(8 \mathrm{~g})$ was dissolved in $100 \mathrm{~mL}$ of $\mathrm{HCl}(9 \mathrm{M})$ in a polytetrafluoroethylene reactor to get a homogenous solution under stirring at room temperature. $\mathrm{After} \mathrm{Ti}_{3} \mathrm{AlC}_{2}(5 \mathrm{~g})$ was added into the $\mathrm{LiF} / \mathrm{HCl}$ solution slowly, the etching reaction lasted for $42 \mathrm{~h}$ under stirring at a constant temperature of $35{ }^{\circ} \mathrm{C}$. The etched product was washed with deionized water by vigorous shaking and centrifuged at $6000 \mathrm{rpm}$ for 5 min to remove the acidic supernatant. The washing-centrifuging process was repeated until the $\mathrm{pH}$ of the dispersion was $\sim 6$. Subsequently, the etched product was diluted with deionized water and centrifugated at $1200 \mathrm{rpm}$ for $20 \mathrm{~min}$ to remove the incompletely etched $\mathrm{Ti}_{3} \mathrm{AlC}_{2}$ or other bulky particles. The supernatant was collected and subjected to the separation procedure one more time. Finally, the supernatant was concentrated by centrifuging at a high speed of $10,000 \mathrm{rpm}$ for $40 \mathrm{~min}$ to obtain a highly concentrated fewlayer $\mathrm{Ti}_{3} \mathrm{C}_{2} \mathrm{~T}_{\mathrm{x}}$ paste with a solid content of approximately 18 wt $\%\left(\sim 196 \mathrm{mg} \mathrm{mL}^{-1}\right)$.

\subsection{Fabrication of Printable MXene/AIOOH Inks}

Firstly, $2.5 \mathrm{~g}$ of the as-prepared MXene paste (18 wt $\%$ ) was diluted with water to $9 \mathrm{wt} \%$ and transferred into a quartz mortar. Subsequently, AlOOH nanoparticles $(100 \mathrm{mg})$ were gradually added into the mortar and the mixture was ground for at least $20 \mathrm{~min}$. After the viscosity of the mixture increased significantly and there was no obvious aggregation of the nanoparticles, the pasty mixture was transferred to a syringe and centrifugated to remove bubbles with a planetary vacuum deaerator at a speed of $1000 \mathrm{rpm}$ for $15 \mathrm{~min}$, and designated as MA3 ink. Similarly, the inks with MXene/ AlOOH mass ratios of 15:1, 10:1 were also prepared and designated as MA1 and MA2, respectively. Table S1 listed the compositions and solid contents of the MA inks. For comparison, pure MXene ink with a solid content of $9 \mathrm{wt} \%$ was also prepared and denoted as pure MX ink.

\subsection{Construction of Frames by Direct Writing with Inks Followed by Etching and Cross-linking}

The syringe was installed on a Jiachuangxing three-axis control direct writing machine (China). The printing pattern was designed by the Autodesk Computer Aided Design (Auto CAD) software and converted to the format of DXF and inputted into the direct writing machine to control the movement path of the syringe. On the basis of the flowability of the MA inks, the printing pressure was fixed at 15-20 psi (pounds per square inch), and the inner diameter of the needle was $410 \mu \mathrm{m}$. The skeletons printed with the MA1, MA2, and MA3 inks were designated as MA1, MA2, MA3, respectively. To remove the $\mathrm{AlOOH}$ component and crosslink the MXene sheets with aluminum ions, the as-printed self-supporting frames were immersed in a solution with $0.5 \mathrm{M} \mathrm{AlCl}_{3}$ and $0.1 \mathrm{M} \mathrm{HCl}$ for $2 \mathrm{~h}$, washed with deionized water to remove the excess $\mathrm{HCI}$ and aluminum ions, and freeze-dried to obtain porous MXene-based frames. The gelled frames by using the inks with MXene/AlOOH mass 
ratios of 15:1, 10:1, and 9:2 were designated as MG1, MG2, and MG3, respectively.

\subsection{Synthesis of Thermochromic PDMS and Assembly with Printed MXene Frames}

Thermochromic polydimethylsiloxane (t-PDMS) was prepared as follows. As the thermochromic component, PCDA was mixed with the prepolymer of PDMS, and polymerized under stirring with the UV light exposure for $1 \mathrm{~min}$. The color change of the mixture from colorless to blue indicates the successful polymerization of PCDA in the prepolymer of PDMS. The polymerized PCDA was also called polydiacetylene (PDA). After the curing agent of PDMS was added, the blue mixture was coated on the surface of the as-printed MG pattern and cured at room temperature to obtain a t-PDMScoated MG pattern.

\subsection{Characterization}

Morphologies of the printed structure were observed with a Hitachi S4700 field emission scanning electron microscope (SEM). Few layered MXene sheets were evaluated by a Hitachi 7700 transmission electron microscope (TEM) and a Bruker multi-mode 8 atomic force microscope (AFM). Rheological behaviors of the inks were tested on an Anton Paar MCR302 rheometer equipped with a 40-mm tape parallel plate geometry $\left(1.985^{\circ}\right.$, cutoff gap of $\left.50 \mu \mathrm{m}\right)$ at $25^{\circ} \mathrm{C}$. Before the rheology tests, all inks were defoamed by centrifuging. Zeta potentials of MXene, $\mathrm{AlCl}_{3} / \mathrm{HCl}$ solution and the aqueous dispersion of $\mathrm{AlOOH}$ were measured with a Malvern Nano-ZS Zetasizer. MXene, MA, and MG were characterized by a Thermo Fisher ESCALAB 250 X-ray photoelectron spectroscope (XPS) and a Rigaku D/Max 2500 X-ray diffractometer (XRD). Electrical conductivities of the 3D printed skeletons were measured with a Four Probes RST-8 resistivity meter (China). The infrared images were obtained using a FLIR-H16 thermal imager (HikVision). EMI shielding performances were measured with a Keysight N5224B PNA series vector network analyzer (VNA) in the frequency range from 8.2 to $12.4 \mathrm{GHz}$. All the samples for the EMI shielding measurements were directly printed to conform the shape of the X-band waveguide cavity.

\section{Results and Discussion}

\subsection{Robust MXene Frames Constructed by Direct Writing with Printable MXene/AlOOH Inks}

Different from conventional EMI shielding materials, future shielding materials are required to satisfy higher integration density and higher design flexibility for more miniaturized and thinner electronic devices. Figure 1a shows the construction of customized porous MXene frames. First, $\mathrm{AlOOH}$ nanoparticles are added into the MXene paste to tune the printability and self-supporting performance, forming uniform MA inks. A pattern can be predesigned with the Auto CAD software, and constructed by a three-axis printing machine with the MA inks. The inks are extruded via a tiny nozzle by external air pressure and form filaments under the movements of the nozzle and the deposition bed. The filaments stack layer by layer with the nozzle lifting in the Z-axis direction to construct a complex 3D architecture (Fig. S1a). Subsequently, the printed MA frames are immersed in $\mathrm{AlCl}_{3} / \mathrm{HCl}$ solution for gelation, during which the $\mathrm{AlOOH}$ nanoparticles are removed on the basis of the reaction between $\mathrm{AlOOH}$ and $\mathrm{HCl}$, and the MXene sheets are cross-linked by the aluminum ions derived from $\mathrm{AlCl}_{3}$ as well as the $\mathrm{AlOOH}$, forming gelled MXene frames. Finally, freeze-drying is adopted to sublime the frozen water and maintain the porous structures of the MXene frames, leading to robust and lightweight MG frames.

Noteworthily, the versatility of DIW technique allows for free design and construction of regular architectures of subwavelength ranges according to specific radio frequencies. One of the prerequisites for printing 3D structures is that the printed filaments could maintain their shapes. For example, the printed ordered cuboids are composed of 20 vertically stacked monofilaments with clear borders and distinct morphology (Fig. 1b, upper). A sub-millimeter porous array with constant filament spacing can be printed readily (Fig. 1b, bottom and Fig. S1c), confirming the capability of the MA ink for clear and fine path designs [34]. By using the MA ink, "open resonant ring" and "commercial EMI shields" are printed as typically shielding architectures (Fig. 1c, d). Moreover, the printed hemispherical frame, "Chinese knots," and pentagrams with conformal sharp angles further confirm 


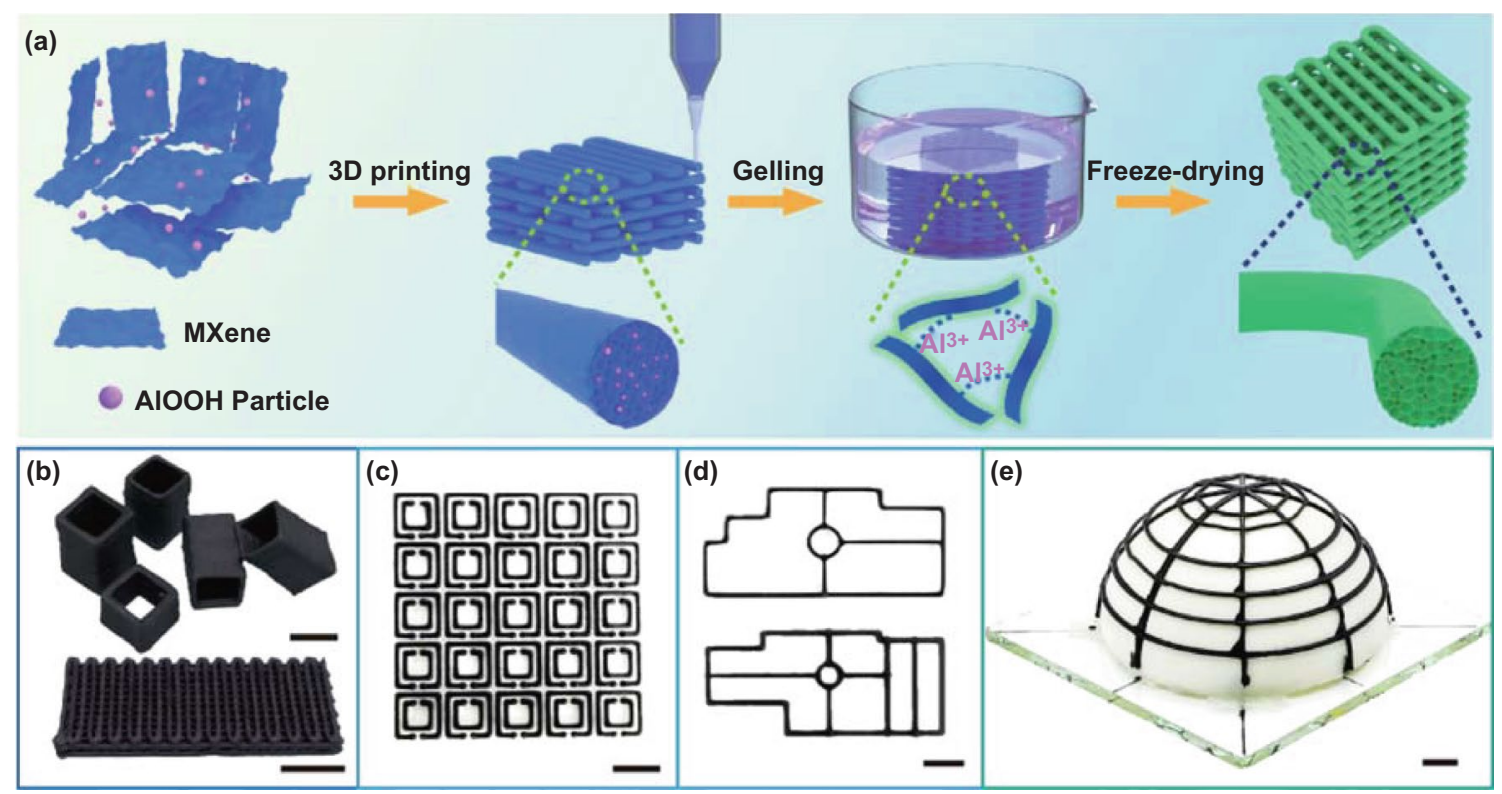

Fig. 1 a Schematic of constructing a freestanding MG frame. b Printed MG cubic frame and "woodpile" microlattice. Printed MG with c double split rings and $\mathbf{d}$ the shape of EMI shields in electronic devices. e Adaptive hemisphere framework by direct ink printing. The scale bars in Fig. 1 b-e is $5 \mathrm{~mm}$

the adaptability of the ink for accurate printing of complex architectures (Figs. 1e and S1b).

As reported, few-layer MXene sheets are prepared by etching the MAX phase precursor with $\mathrm{HCl} / \mathrm{LiF}$ solution, followed by delamination under vigorous hand-shaking. After etching, the XRD peak downward shifts from $9.5^{\circ}$ to $6.3^{\circ}$, corresponding to the increase of interlayer spacing from 9.3 to $14.0 \AA$ due to the intercalation of lithium ions and water molecules (Fig. S2a) [35]. Consistent with the greatly weakened (104) peak, the exfoliated MXene sheets are transparent with crumpled edges, indicating the ultrathin and flexible features (Fig. S2b). The MXene sheets are 1-4 $\mu \mathrm{m}$ in lateral sizes on the basis of the statistics of TEM images (Fig. S2c) and 3-5 nm in thickness (Fig. S2d), exhibiting a large aspect ratio and few structural defects.

Analogous to long-chain polymers, a dispersion of MXene sheets with a large aspect ratio usually exhibits high viscosity because of the sheet-to-sheet resistance under external shear forces [36]. The viscoelastic properties of an MXene dispersion can be adjusted by varying the MXene content to satisfy the different processing techniques [37], i.e., screen printing [38], spraying [39], and extrusion printing [40]. Generally, viscosities of the inks for directly writing should be in the range from 0.1 to $10^{3} \mathrm{~Pa}$ s [41]. Figure S3a shows apparent viscosities of pure MX ink and MA inks.
The high viscosities of the inks at low shear rates indicate their solid-like state. Compared with MA2 ink, MA1 ink has a higher viscosity because of the higher content of MXene (Table S1). Although MA3 ink has the same overall solid content as MA2 ink, the former has a higher ratio of $\mathrm{AlOOH}$ nanoparticles. The increased viscosity of MA3 is attributed to the thickening effect of the $\mathrm{AlOOH}$ nanoparticles. The thickening effect of the $\mathrm{AlOOH}$ viscosifier is more obvious by comparing the viscosities of the MA3 ink and pure MX ink [42]. As the shear rate increases, the viscosity of all samples decreases rapidly, showing typical shear thinning characteristics. The effect of $\mathrm{AlOOH}$ nanoparticles on viscoelasticity is also confirmed by the oscillation amplitude tests (Fig. S3b). The storage modulus and loss modulus of the MA3 ink have an order of magnitude increase than that of pure MX ink. This indicates that the $\mathrm{AlOOH}$ nanoparticles increase rigidity and flow resistance of the ink, which would be beneficial for shape retention of the printed filaments [43]. All the MA inks have large plateau regions at initial shear stresses and their storage moduli are much higher than their loss moduli (G'> G' '), presenting solid-like behaviors. When the shear stress approaches the yield stress, both the moduli drop sharply and viscous deformations occur (G'<G') (Fig. S3c). Compared with MA1 ink, the modulus of MA3 ink increases more obviously, indicating that the 
addition of $\mathrm{AlOOH}$ nanoparticles enhances the resistance of ink to external force deformation. These results suggest that optimal viscoelastic properties of MA inks can satisfy the requirements for direct writing and the MG frames with different properties can be obtained by adjusting components of MA inks.

\subsection{Enhanced Mechanical and Electrical Performances of MXene Frames Reinforced by Cross-linking of MXene Sheets with Aluminum Ions}

To construct robust and porous MXene-based frames, the printed MA frames are immersed in $\mathrm{HCl} / \mathrm{AlCl}_{3}$ solution to remove the electrically insulating $\mathrm{AlOOH}$ nanoparticles and to cross-link the MXene sheets by the aluminum ions. The dissolution of $\mathrm{AlOOH}$ with $\mathrm{HCl}$ generates numerous voids inside the filaments after freeze-drying, which would decrease the density of the printed frames. The in situ generated aluminum ions along with those of $\mathrm{AlCl}_{3}$ can cross-link the MXene sheets during the gelation process, reinforcing the filaments and enhancing joint between them. Figure 2 shows the morphology and microstructures of the wood-pile MG frames. Clearly, the individual filaments present welldefined uniform and distinct linear shapes without dislocation or fracture (Fig. 2a). Typically, the monofilament shows a diameter of $400 \pm 30 \mu \mathrm{m}$, indicating the stable thixotropy and the self-supporting properties of the MA ink (Fig. 2b). The side-view (Fig. 2c) and cross-sectional view (Fig. 2d) SEM images show that the longitudinal filaments are arranged at equal intervals on the horizontal filaments. The crisscross filaments closely interconnect with each other to form continuous regular macroscopic architecture with jointed interfaces. In addition, the quadrilateral pores can be adjusted from millimeter to sub-millimeter by varying the filament spacing.

Moreover, the cross-section observation reveals the microporous feature of the MG frames (Fig. 2e, f). The fracture surface of filaments exhibits nearly circular appearances with densely packed micropores of 10 to $30 \mu \mathrm{m}$, which are attributed to the in situ subliming of the grown ice crystals during the freeze-drying process (Fig. 2f). Different from the random micropores for the MA frame (Fig. S4a, b), the "cabbage-like" microcellular structure of the crosslinked MG is more compact with better interconnection and continuity because of the gelation effect of aluminum ions (Fig. 2g). Furthermore, the abundant aluminum ions can interact with the dangling hydroxyl groups of MXene sheets to form a robust internal network (Fig. 2h). On the contrary, in the absence of the reinforcement of aluminum ions, the MXene sheets are weakly interconnected and disrupted by the growth of ice crystals, forming a random and fragile porous microstructure (Fig. S4c, d). MG1 and MG3 also exhibit "cabbage-like" microcellular structures due to the interaction of the aluminum ions with the MXene sheets (Fig. S5). The pores in the MG3 frame are more obvious than that of MG2 due to the lower density of MG3 (Table S2). A denser structure exists in the MG1 filaments because of its higher MXene content. The MXene sheets in all these frames are linked tightly by the aluminum ions to form the sinuous inner structures. Therefore, the introduced AlOOH nanoparticles not only improve the printability of the inks during the printing process, but also produce aluminum ions to in situ cross-link the MXene sheets, benefiting the structural stability of the printed frames even with low bulk densities (Table S2).

To figure out the reinforcement mechanism of aluminum ions, the printed MA3 frames are immersed in water, $0.1 \mathrm{M}$ $\mathrm{HCl}$ solution, $0.5 \mathrm{M} \mathrm{AlCl}_{3}$ solution, and $0.5 \mathrm{M} \mathrm{AlCl}_{3} / 0.1 \mathrm{M}$ $\mathrm{HCl}$ solution (Fig. S6). Clearly, the printed MA frames are readily collapsed after the immersion and washing processes in the absence of $\mathrm{AlCl}_{3}$. It means that the in situ generated aluminum ions by the etching of $\mathrm{AlOOH}$ are not sufficient for cross-linking all the MXene sheets to sustain the whole macroscopic 3D architecture. Besides, the weak interactions between the MXene sheets are not capable to resist the rehydration effect [44]. In contrast, the presence of $\mathrm{AlCl}_{3}$ provides sufficient aluminum ions to offer favorable interfacial interconnection and coalescence between the filaments (Fig. S6c) [45]. When the MA frame is immersed in the $\mathrm{AlCl}_{3} /$ $\mathrm{HCl}$ solution, the printed frame keeps its customized structure because of the cross-linking effect with aluminum ions, and the removal of the $\mathrm{AlOOH}$ component is achieved simultaneously (Fig. S6d). These results confirm the reinforcement of printed architectures by the cross-linking with aluminum ions. 

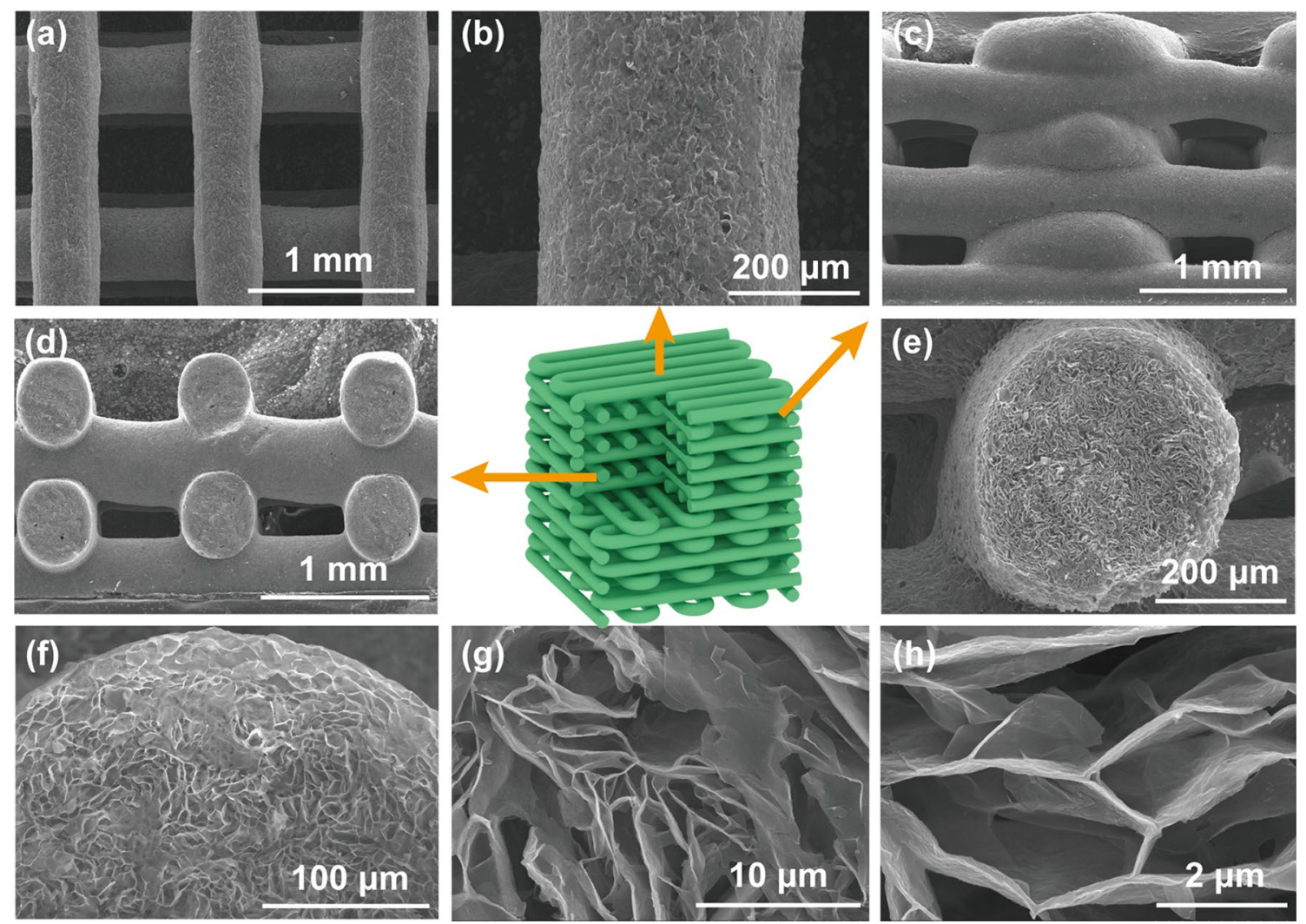

Fig. 2 SEM images of porous MG2 with a wood-pile structure from different directions: a, b top view, c side view, and d, e cross-sectional view. $\mathbf{f}-\mathbf{h}$ Cross-sectional SEM images of MG2 cross-linked by aluminum ions

As the MXene suspension presents negative zeta potential $(-27 \mathrm{mV})$ derived from the negative functional group of MXene sheets (Fig. S7a), the positive aluminum ions could link these sheets via electrical interactions. This phenomenon is further confirmed by the TEM image of the MXene sheets extracted from an MG3 frame by sonication (Fig. 3a). The evenly distributed $\mathrm{AlCl}_{3}$ crystals between the closely stacked MXene sheets indicate the strong interaction of aluminum ions with adjacent MXene sheets. The XPS spectra are shown in Fig. S7b. Consistently, as compared to MA3, MG3 presents an enhanced $\mathrm{Al} 2 \mathrm{p}$ peak and a stronger peak of Al-O bond at $532.5 \mathrm{eV}$ (Figs. $3 \mathrm{~b}$ and S7c), indicating the bonding of aluminum ions with oxygen-containing surface groups of MXene. In the F $1 \mathrm{~s}$ spectrum (Fig. 3c), the amplified Al-F peak is another evidence for the combination of aluminum ions with the negatively charged MXene sheets [46]. The Ti 2p XPS and Raman spectra of MA3 and MG3 do not have large changes, suggesting the maintaining of intrinsic properties of MXene during the gelation process [47] (Fig. 3d, e). The intercalation of aluminum ions between the MXene sheets is also verified by the downward shifted (002) peak of MG3 relative to that of MXene [48]. This phenomenon is also observed in the MG1 and MG2 frames due to the shift of (002) peaks in their XRD curves (Fig. S8a, b). The same elements and surface groups of MG1 and MG2 imply that the intrinsic properties of MXene are well kept after the cross-linking (Fig. S8c, d). In a word, as shown in Fig. $3 \mathrm{~g}$, the trivalent aluminum ions act as a linker to interconnect the MXene sheets and hence enhance their interfacial interaction.

The reinforcement of aluminum ions greatly improves the structural stability of the frame to resist external deformation. It can withstand a weight of almost 3000 times its own weight (Fig. S9a). Also, the aluminum ion-reinforced robust structure of MG3 enables it to resist ultrasonication for $2 \mathrm{~min}$ in water (Fig. S9b), as evidenced by the intact 

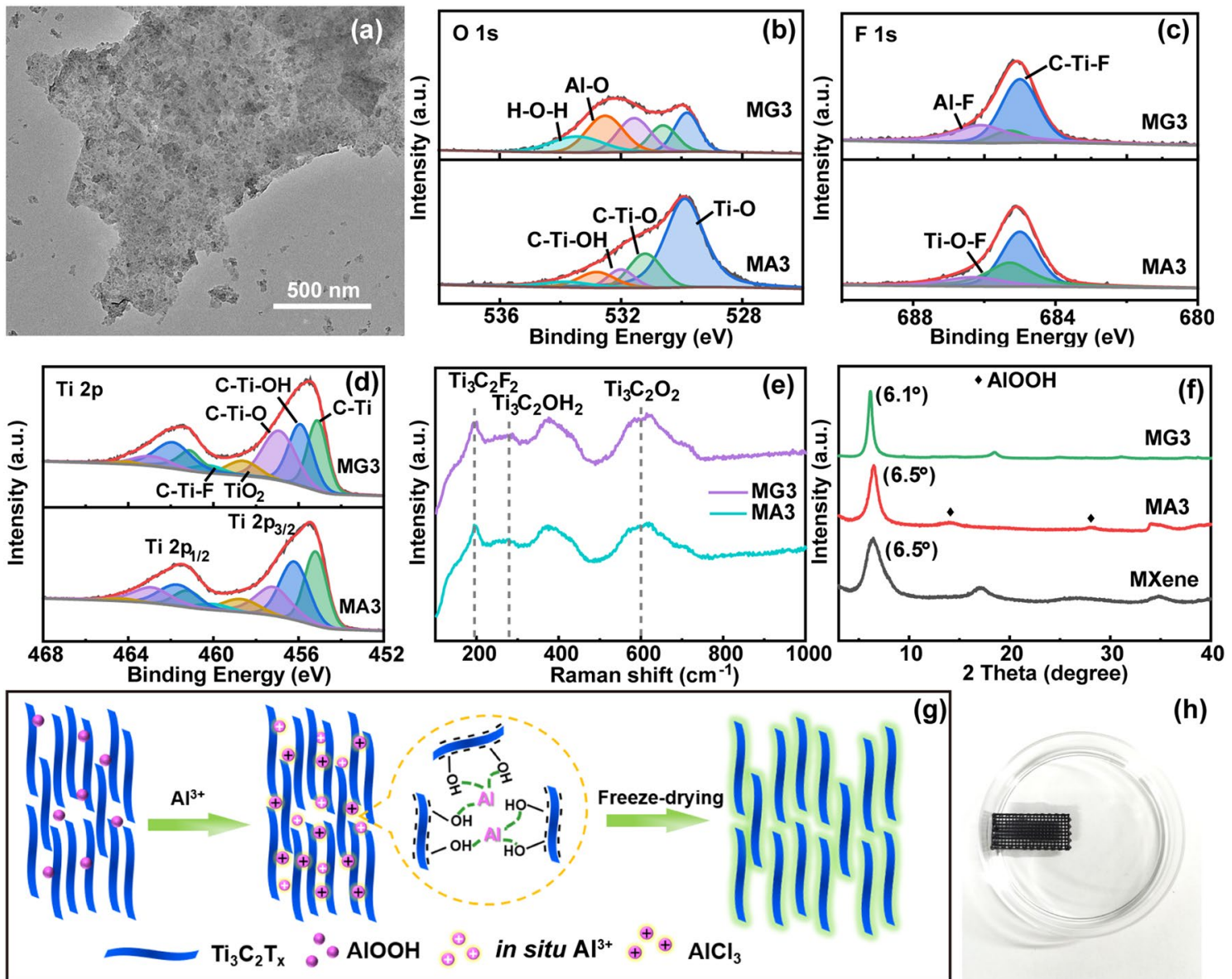

(h)

Fig. 3 a TEM image of cross-linked MXene sheets extracted from a MG frame. b O 1 s, c F 1 s, and d Ti 2p XPS spectra of MA3 and MG3. e Raman spectra of MA3 and MG3. f XRD patterns of MXene, MA3, and MG3. $\mathbf{g}$ Aluminum ion-induced gelation of MXene sheets. $\mathbf{h}$ Intact MG3 frame after ultrasonication

architecture of the MXene frame in water after the ultrasonication (Fig. 3h). The mechanical measurements further reveal the strengthening effect of the aluminum ions on the printed frames (Fig. S10). The compression strengths of MG frames are higher than those of their corresponding MA frames. Although there are plateau regions in the MA curves, the internal structures of MA frames are already destroyed when the strains reach their yielding points. The compression strength of MG3 is $186 \mathrm{kPa}$, much higher than that of MA3 (84 kPa). Similarly, MG2 has a higher compression strength of $241 \mathrm{kPa}$ than that of MA2 $(98 \mathrm{kPa})$. MG1 has the highest compression strength of $268 \mathrm{kPa}$, which is attributed to its highest density. All these results verify the reinforcement effect of the aluminum ions on the MG frames. The in situ generated aluminum ions from the $\mathrm{AlOOH}$ and the added aluminum ions from $\mathrm{AlCl}_{3}$ could well reinforce the interaction between MXene sheets inside the filaments and between the filaments, while maintaining the inherent conductive properties of MXene, which would ensure satisfactory EMI shielding performances of the MXene frames [49].

\subsection{Electrically Conductive and EMI Shielding Performances of Robust and Porous MXene Frames}

Thanks to the cross-linking of $\mathrm{Al}^{3+}$ ions, the MG frame fabricated by the versatile DIW method exhibits continuous interconnection between filaments with well jointed crisscross interfaces (Fig. 4a, b), endowing the lightweight and integrated hierarchical architectures with outstanding electrical conductivities. MG3 has an electrical conductivity of up to $4119 \mathrm{~S} \mathrm{~m}^{-1}$ with a bulk density of $71 \mathrm{mg} \mathrm{cm}^{-3}$, and 
the highest conductivity of 5,323 $\mathrm{S} \mathrm{m}^{-1}$ is achieved for MG1 with a large MXene content (Fig. 4c). The high conductivity of the printed frame is well reflected by its electromagnetic induction experiment. For example, a LED bulb connected to the printed MG filament circuit can be lightened by the alternating magnetic field supplied by the induction cooker (Figs. 4d and S11, Video S1). Particularly, the generated alternating magnetic field can stimulate electrons of MXene sheets to freely transport through the conductive filaments, and lighten the LED light. The LED bulb can work normally if the magnetic field intensity is high enough. This result proves the good continuity and high conductivity of the printed MG filament circuit.
Furthermore, the versatile DIW technique can produce EMI shielding materials with predesigned structures for complex electronic packaging applications without posttreatment. For example, EMI shielding samples that are exactly adaptive to the waveguide cavity can be printed, and their shielding performances can be tuned finely by adjusting the printing layer number, filament constituents, and spacing. With a constant filament spacing of $1 \mathrm{~mm}$ and a thickness of $\sim 2 \mathrm{~mm}$, the overall EMI shielding performance is improved with increasing the MXene content in the filament (Fig. 4e), which is consistent with the increased tendency of electrical conductivity of the printed frames. MG3 delivers an average EMI SE value of $43.5 \mathrm{~dB}$, which can block $99.99 \%$ of the incident electromagnetic waves. Among the
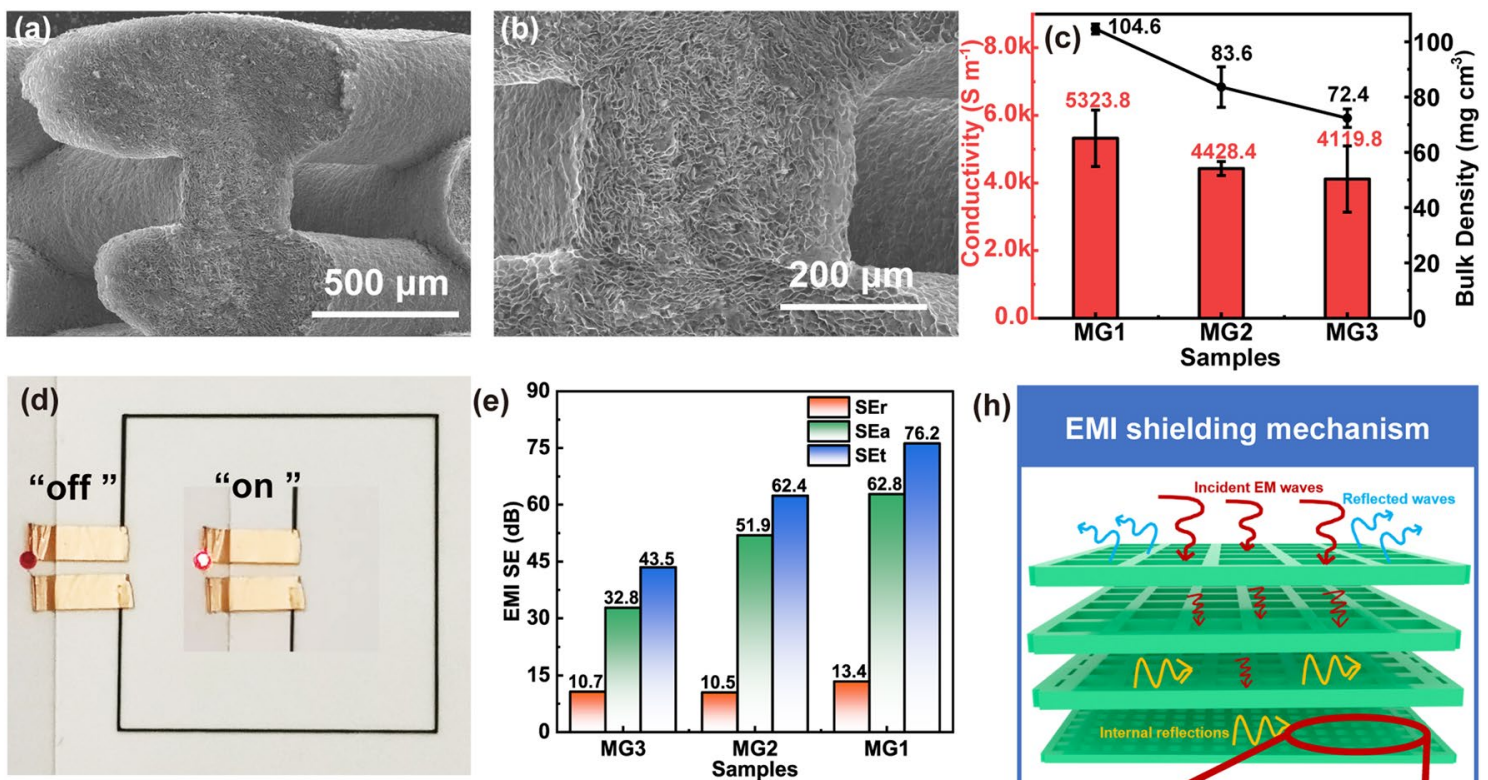

(h)

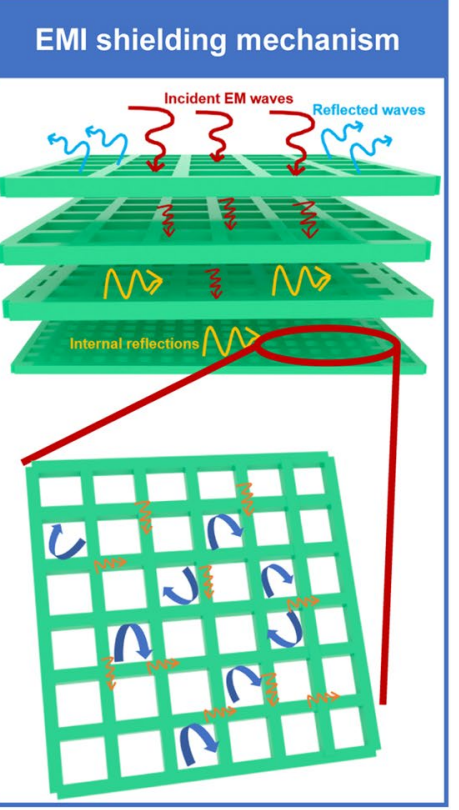

Fig. 4 a, b SEM images of interfacial connection of MG2 frame. c Electrical conductivities and bulk densities of MG1, MG2, and MG3 frames with a constant filament spacing of $1 \mathrm{~mm}$. d LED blub is lightened with an MG frame under an alternating electromagnetic field. e SEt, SEr, and SEa values of MG1, MG2 and MG3 frames with different filament constituents. The thickness is $2 \mathrm{~mm}$ and the filament spacing is $1 \mathrm{~mm}$. $\mathbf{f}$ EMI shielding performances of MG1 with different filament spacings. $\mathbf{g}$ Influences of printing layer on the EMI shielding performances of MG1, MG2, and MG3 frames. h EMI shielding mechanisms of MG frame 
explored samples, MG1 exhibits the highest EMI SE of $76.2 \mathrm{~dB}$, superior to most of the 3D printing EMI shielding materials reported (Table S3). In addition, all these MXene frames show an absorption-dominant EMI shielding mechanism. By changing the filament spacing of MG1 from 1.5 to $1.0 \mathrm{~mm}$, the average EMI shielding effectiveness (SE) increases from 62.7 to $71.5 \mathrm{~dB}$ (Fig. 4f). The normalized surface specific EMI SE of MG1-1 mm reaches $5044.1 \mathrm{~dB}$ $\mathrm{cm}^{2} \mathrm{~g}^{-1}$. Noticeably, further reduction in spacing to $0.5 \mathrm{~mm}$ affords an outstanding shielding performance of more than $80 \mathrm{~dB}$ over the entire X-band frequency range at a small thickness of $1.37 \mathrm{~mm}$, and the normalized thickness specific SE is over $61.0 \mathrm{~dB} \mathrm{~mm}^{-1}$, exhibiting the advantage of the DIW fabrication of 3D electromagnetic shielding materials.

The frame thickness can be freely adjusted by controlling the printed layer number. The MG frames with 3, 4, and 6 layers are $\sim 0.75, \sim 1.35$, and $\sim 2 \mathrm{~mm}$ in thickness, respectively. With the layer number changes, the EMI shielding performances can be tuned in the range of $25-76 \mathrm{~dB}$ (Fig. 4g). The excellent shielding performances can be ascribed to the high conductivity of the MG frame and its hierarchical porous structures with abundant interfaces [50].

The pore size of the printed frames also affects the EMI shielding performances [51]. As reported, the shielding performance in the X-band would be largely affected when the pore length is over $1.8 \mathrm{~mm}$ [52]. Fortunately, the square hole of the MG frame with a spacing of $1.5 \mathrm{~mm}$ is $\sim 1.1 \mathrm{~mm}$, smaller than one-tenth of the shortest wavelength of X-band electromagnetic waves and sufficient to block the free penetration of electromagnetic waves. Figure $4 \mathrm{~h}$ shows the attenuation mechanism of electromagnetic waves by an MG frame. When the electromagnetic wave strikes the frame surface, a large part is reflected because of the high electron carrier density of the conductive MG frame. The incident wave is repeatedly scattered and diffracted inside the hierarchical porous structure, greatly extending the wave propagation path and leading to effective energy dissipation [53, 54]. In addition, the electromagnetic wave-activated electrons in the MG frame can migrate along the conductive filaments and jump across the interfaces or defects, generating microcurrents inside the filaments that would be beneficial for absorbing and dissipating the electromagnetic energy in the form of thermal energy $[55,56]$.

\subsection{Assembly of Thermochromic MG Patterns for Electromagnetic-Thermochromism and Electro-Thermochromism Applications}

Inspired by the conversion of electromagnetic energy to thermal energy, electromagnetic wave-induced thermochromic (EWT) patterns are developed by assembling the printed MG3 patterns with a thermochromic polymer of t-PDMS. Interestingly, high-intensity radiation field (HIRF) can be seen directly by our naked eyes because of the color change of the thermochromic MG patterns. This distinct color change derives from the conformational change of the PDA component of t-PDMS [57]. Stimulated by the heat generated by the irradiation of MG patterns with the strong alternating electromagnetic waves, the side-chain strains of PDA tend to release the mechanical strain generated by its polymerization. The side-chain distortion disturbs the p-orbital arrays to induce the color change from blue to red. The colorimetric response (CR) value calculated by the absorbance of t-PDMS in initial and final states can reach $40 \%$ (Figs. $5 \mathrm{~b}$ and S13a) [58]. Apparently, t-PDMS exhibits a distinct color transition from blue to red with its temperature increases from 25 to $70{ }^{\circ} \mathrm{C}$.

As shown in Fig. 5a, the "electromagnetic wave" pattern of MG3 is printed by the DIW technique using the MA3 ink on a glass base. After t-PDMS is coated and cured, the thermochromic MG3 is put in a microwave oven with a working frequency of $2.45 \mathrm{GHz}$, which can provide a strong alternating electromagnetic wave radiation field of $50 \mathrm{~W}$ (Fig. S12). Upon irradiation, the PDA directly contacted with MG3 quickly changes its color from blue to red within $3 \mathrm{~s}$. The red color becomes more obvious when the thermochromic MG3 pattern is irradiated for another $2 \mathrm{~s}$, indicating its temperature might be over $70{ }^{\circ} \mathrm{C}$ based on the color change of t-PDMS at different temperatures (Fig. 5b, c). However, the rest area of the t-PDMS that does not contact the MG3 pattern still maintains its original blue color. This phenomenon demonstrates that electromagnetic waves cannot cause the color change of t-PDMS itself. The thermal energy generated by interacting the alternating electromagnetic waves with the printed MG3 pattern is the origin of the color change of t-PDMS [59]. In addition, the filament interfaces 

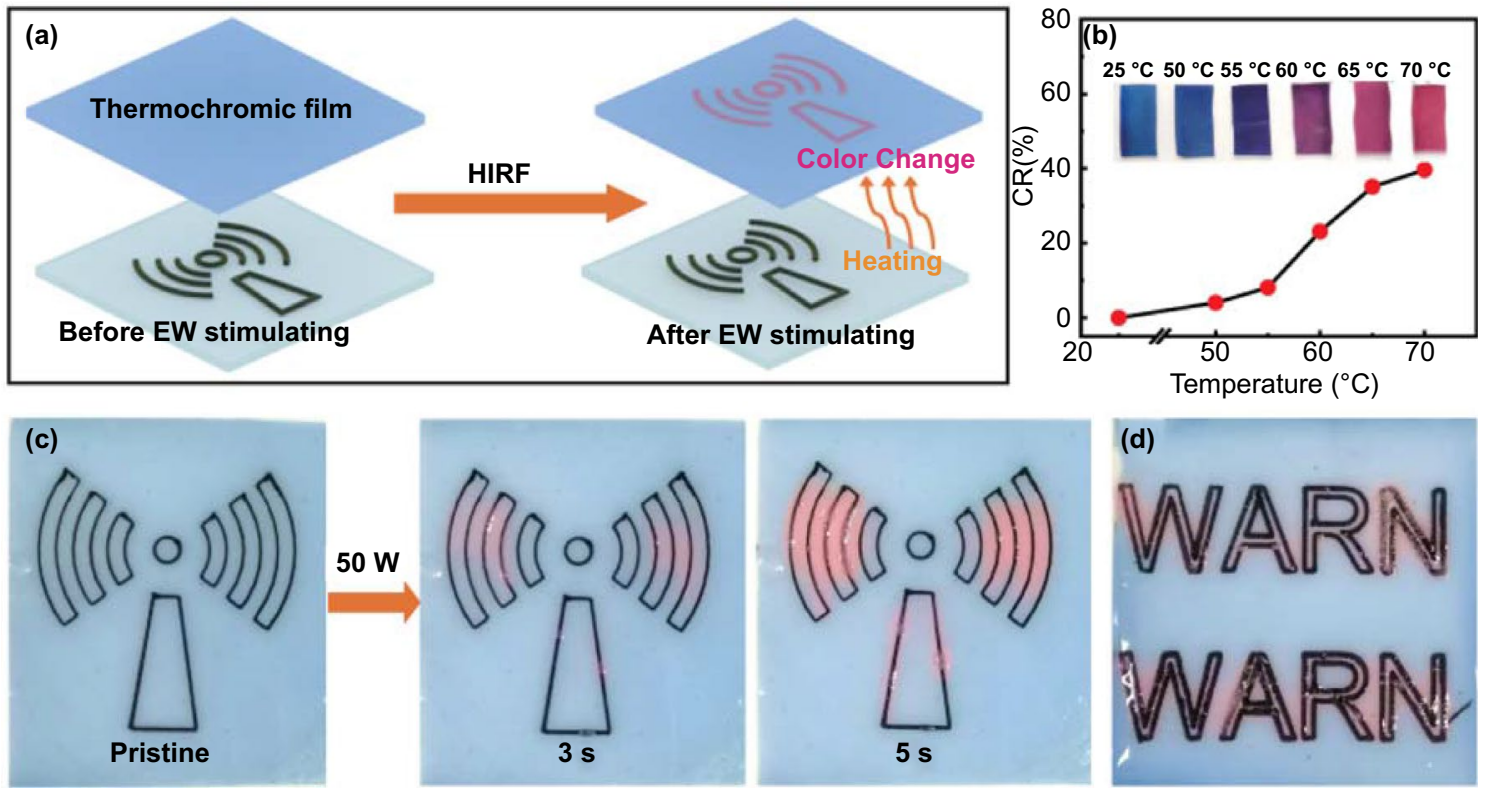

Fig. 5 a Schematic of the fabrication of high-intensity radiated field (HIRF) responsive materials. b Colorimetric response (CR) values of t-PDMS from 25 to $70{ }^{\circ} \mathrm{C}$. c, d Photographs of thermochromic MG3 patterns stimulated by electromagnetic waves of $2.45 \mathrm{GHz}$

or defects may cause charge accumulation and induce sparkle light, further confirming the severe interaction of MG3 with electromagnetic waves in the strong electromagnetic radiation field. In principle, the high-intensity alternating electromagnetic field enables charge carriers to migrate in a continuous printed pattern pathways [60]. The resultant induced current generates heat via vibration and migration of electrons, and thereby converts the invisible microwave to visible forms via the reaction-triggered color variation [61]. The shape of the MG pattern can also be designed as the word "WARN" (Fig. 5d), which could remind people to protect against high-intensity electromagnetic waves by the color change stimulated by strong alternating electromagnetic wave radiation fields. Based on these results, it is reasonable to design more sensitive EWT composites that can detect low-intensity electromagnetic waves by synthesizing low-temperature responsive thermochromic polymers. The visualization of electromagnetic waves is promising for potential warning of daily electromagnetic radiation, highintensity electromagnetic pulse, and other related fields.

In addition to the electromagnetic wave-induced thermochromism, the electro-thermochromism of the thermochromic MG patterns is also proved by settling in a direct current source [62]. The temperature-sensitive color variation can act as an apparently visible indicator to in situ reflect the real temperature and voltage. Figure 6a shows the physical image of an electro-thermochromic MG3 pattern. The color change from blue to red directly indicates the excessively high temperature and applied voltage. With the MG3 frame as the Joule heater, the good linear relationship between the saturation temperature and the square of the voltage conforms well with the fitted curve, making it possible to finely control the target temperature with the applied voltage (Fig. 6b) [63]. Interestingly, the high conductivity of the printed MG3 pattern even affords a high temperature of $105^{\circ} \mathrm{C}$ under a low voltage of only $1.5 \mathrm{~V}$.

Figure $6 \mathrm{c}$ shows the temperature dependence of the MG3 pattern under different voltages, exhibiting good heating-cooling ability. As the applied voltage increases, the heating rate and the saturation temperature are greatly elevated. At $1.25 \mathrm{~V}$, the temperature rises from 23 to $83{ }^{\circ} \mathrm{C}$ within $160 \mathrm{~s}$ and then decreases to room temperature after $140 \mathrm{~s}$ once the voltage is turned off, presenting a good heating-cooling recovery ability. A stable temperature of $42{ }^{\circ} \mathrm{C}$ can be kept at only $0.75 \mathrm{~V}$, satisfying the requirement for keeping warm or relieving pain and stiffness of muscles. Figure $6 \mathrm{~d}$ shows the time-dependent surface temperatures under different voltages, further confirming the low-voltage joule heating performance of the printed MG circuit. The temperature increase can be seen from the infrared thermal imager, while it also can be figured out by the color change from blue to red of the MG3 pattern (Fig. 6e, f). 

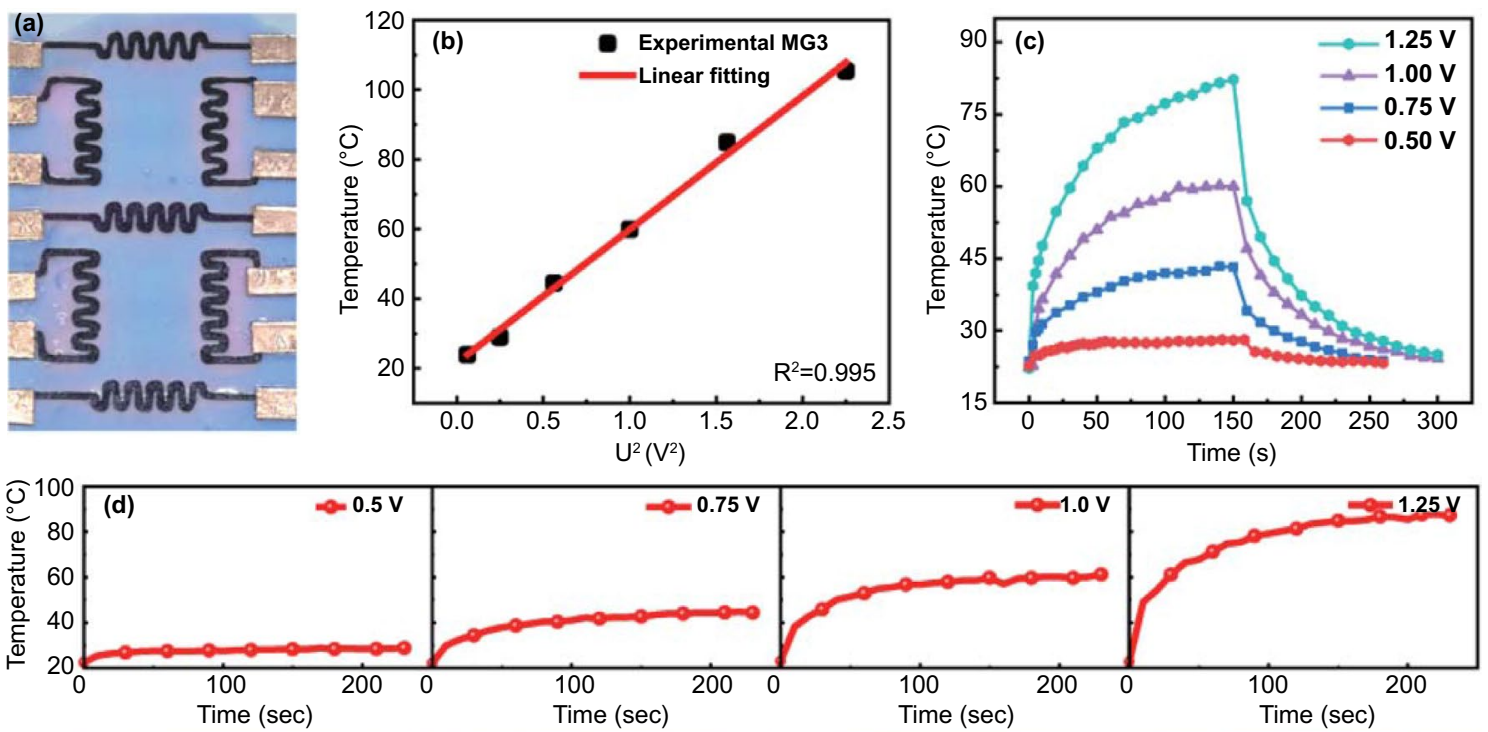

(e)
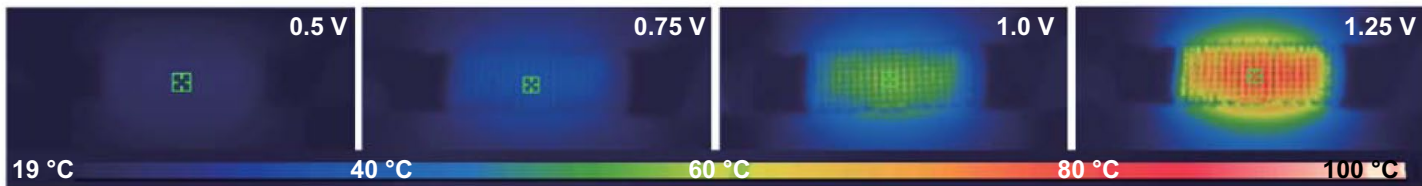

(f)

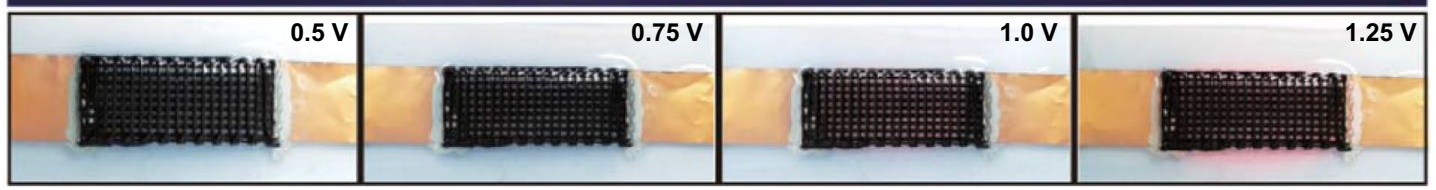

Fig. 6 Electric thermal performances of printed MG3. a Digital photograph of printed thermochromic pattern. b Relationship between saturation temperature and $\mathrm{U}^{2}$ for MG3 pattern. c Time-dependent surface temperatures under different voltages. d Joule heating performances of MG3 pattern, e infrared thermal imaging photographs, and $\mathbf{f}$ color changes of electro-thermochromic MG3 pattern under related voltages

The electro-thermochromic ability of thermochromic MG patterns provides a way to recognize excessive temperature by naked eyes. Moreover, the MG3 pattern also has excellent long-term stability and recyclability, as evidenced by the stable temperature of $60{ }^{\circ} \mathrm{C}$ under $1 \mathrm{~V}$ (Fig. S13b). All these results verify that the thermochromic MG patterns can also be utilized to assemble smart electric heating devices with color change when the temperature is excessively high.

\section{Conclusions}

Robust and highly conductive MXene frames with customized structures are fabricated by a direct ink writing technique with printable MXene/AlOOH inks for tunable electromagnetic interference shielding and electromagneticthermochromic applications. The 3D frames with predesigned structures are readily printed by combining MXene with $\mathrm{AlOOH}$ nanoparticles to form printable inks and then immersed in $\mathrm{HCl} / \mathrm{AlCl}_{3}$ mixture to remove the electrically insulating $\mathrm{AlOOH}$ nanoparticles and reinforce the MXene architecture by cross-linking of MXene sheets with aluminum ions inside and between the printed filaments. After freeze-drying to subliming the frozen water, the resultant porous and robust MG frame can sustain a weight almost 3000 times of its weight, also exhibits a high electrical conductivity of $5,323 \mathrm{~S} \mathrm{~m}^{-1}$ and a satisfactory EMI shielding efficiency of $80 \mathrm{~dB}$ in the whole X-band. The surface specific SE achieves $5,044.1 \mathrm{~dB} \mathrm{~cm}^{2} \mathrm{~g}^{-1}$ when MG has a thickness of $1.35 \mathrm{~mm}$ and a bulk density of $0.105 \mathrm{~g} \mathrm{~cm}^{-3}$. The EMI shielding efficiency can be tuned in the range of 25 to $80 \mathrm{~dB}$ by adjusting the printing parameters. Furthermore, by assembling the printed MG patterns with a thermochromic t-PDMS synthesized, the resulting thermochromic MG patterns exhibit a distinct color change from blue to red on the basis of electromagnetic-thermochromism and electro-thermochromism, which is driven by the heat converted from 
electromagnetic energy and electric energy. The assembled thermochromic MXene-based patterns are promising in the fields of electromagnetic anti-counterfeit labels, strong electromagnetic radiation warning, electromagnetically or electrically converted thermal management equipment.

Acknowledgements Financial support from the National Natural Science Foundation of China (51922020, 52090034), and the Fundamental Research Funds for the Central Universities (BHYC1707B) is gratefully acknowledged.

Open Access This article is licensed under a Creative Commons Attribution 4.0 International License, which permits use, sharing, adaptation, distribution and reproduction in any medium or format, as long as you give appropriate credit to the original author(s) and the source, provide a link to the Creative Commons licence, and indicate if changes were made. The images or other third party material in this article are included in the article's Creative Commons licence, unless indicated otherwise in a credit line to the material. If material is not included in the article's Creative Commons licence and your intended use is not permitted by statutory regulation or exceeds the permitted use, you will need to obtain permission directly from the copyright holder. To view a copy of this licence, visit http://creativecommons.org/licenses/by/4.0/.

Supplementary Information The online version contains supplementary material available at https://doi.org/10.1007/ s40820-021-00665-9.

\section{References}

1. M.S. Cao, X.X. Wang, M. Zhang, J.C. Shu, W.Q. Cao et al., Electromagnetic response and energy conversion for functions and devices in low-dimensional materials. Adv. Funct. Mater. 29(25), 1807398 (2019). https://doi.org/10.1002/adfm.20180 7398

2. F. Shahzad, M. Alhabeb, C.B. Hatter, B. Anasori, S.M. Hong et al., Electromagnetic interference shielding with 2d transition metal carbides (MXenes). Science 353(6304), 1137-1140 (2016). https://doi.org/10.1126/science.aag2421

3. R. Yang, X. Gui, L. Yao, Q. Hu, L. Yang et al., Ultrathin, lightweight, and flexible CNT buckypaper enhanced using mxenes for electromagnetic interference shielding. Nano-Micro Lett. 13(1), 66 (2021). https://doi.org/10.1007/s40820-021-00597-4

4. Y. Zhang, K. Ruan, X. Shi, H. Qiu, Y. Pan et al., $\mathrm{Ti}_{3} \mathrm{C}_{2} \mathrm{~T}_{\mathrm{x}} / \mathrm{rGO}$ porous composite films with superior electromagnetic interference shielding performances. Carbon 175, 271-280 (2021). https://doi.org/10.1016/j.carbon.2020.12.084

5. W.T. Cao, C. Ma, D.S. Mao, J. Zhang, M.G. Ma et al., MXenereinforced cellulose nanofibril inks for 3D-printed smart fibres and textiles. Adv. Funct. Mater. 29(51), 1905898 (2019). https://doi.org/10.1002/adfm.201905898

6. L.X. Liu, W. Chen, H.B. Zhang, Q.W. Wang, F. Guan et al., Flexible and multifunctional silk textiles with biomimetic leaf-like MXene/silver nanowire nanostructures for electromagnetic interference shielding, humidity monitoring, and self-derived hydrophobicity. Adv. Funct. Mater. 29(44), 1905197 (2019). https://doi.org/10.1002/adfm.201905197

7. X. Wu, B. Han, H.B. Zhang, X. Xie, T. Tu et al., Compressible, durable and conductive polydimethylsiloxane-coated MXene foams for high-performance electromagnetic interference shielding. Chem. Eng. J. 381, 122622 (2020). https://doi. org/10.1016/j.cej.2019.122622

8. P. Song, B. Liu, H. Qiu, X. Shi, D. Cao et al., MXenes for polymer matrix electromagnetic interference shielding composites: a review. Compos. Commun. 24, 100653 (2021). https://doi.org/10.1016/j.coco.2021.100653

9. A. Iqbal, P. Sambyal, C.M. Koo, 2D MXenes for electromagnetic shielding: a review. Adv. Funct. Mater. 30(47), 2000883 (2020). https://doi.org/10.1002/adfm.202000883

10. X.Y. Wu, H.B. Zhang, Study on structure design and electromagnetic shielding properties of polymer nanocomposites. Acta Polym. Sin. 51(6), 573-583 (2020). https://doi.org/10. 11777/j.issn1000-3304.2020.20015

11. Z. Zeng, C. Wang, G. Siqueira, D. Han, A. Huch et al., Nanocellulose-MXene biomimetic aerogels with orientationtunable electromagnetic interference shielding performance. Adv. Sci. 7(15), 2000979 (2020). https://doi.org/10.1002/advs. 202000979

12. P. Song, B. Liu, C. Liang, K. Ruan, H. Qiu et al., Lightweight, flexible cellulose-derived carbon aerogel@reduced graphene oxide/PDMS composites with outstanding EMI shielding performances and excellent thermal conductivities. Nano-Micro Lett. 13(1), 91 (2021). https://doi.org/10.1007/ s40820-021-00624-4

13. Y. Ma, Y. Yue, H. Zhang, F. Cheng, W. Zhao et al., 3D synergistical MXene/reduced graphene oxide aerogel for a piezoresistive sensor. ACS Nano 12(4), 3209-3216 (2018). https:// doi.org/10.1021/acsnano.7b06909

14. Y. Dai, X. Wu, Z. Liu, H.B. Zhang, Z.Z. Yu, Highly sensitive, robust and anisotropic MXene aerogels for efficient broadband microwave absorption. Compos. B: Eng. 200, 108263 (2020). https://doi.org/10.1016/j.compositesb.2020.108263

15. C. Weng, G. Wang, Z. Dai, Y. Pei, L. Liu et al., Buckled AgNW/MXene hybrid hierarchical sponges for high-performance electromagnetic interference shielding. Nanoscale 11(47), 22804-22812 (2019). https://doi.org/10.1039/c9nr0 7988 b

16. S. Shi, B. Qian, X. Wu, H. Sun, H. Wang et al., Self-assembly of MXene-surfactants at liquid-liquid interfaces: from structured liquids to 3D aerogels. Angew. Chem. Int. Ed. 58(50), 18171-18176 (2019). https://doi.org/10.1002/anie.201908402

17. R. Sun, H.B. Zhang, J. Liu, X. Xie, R. Yang et al., Highly conductive transition metal carbide/carbonitride(MXene)@ polystyrene nanocomposites fabricated by electrostatic assembly for highly efficient electromagnetic interference shielding. Adv. Funct. Mater. 27(45), 1702807 (2017). https://doi.org/10. 1002/adfm.201702807

18. N. Zhou, C. Liu, J.A. Lewis, D. Ham, Gigahertz electromagnetic structures via direct ink writing for radio-frequency 
oscillator and transmitter applications. Adv. Mater. 29(15), 1605198 (2017). https://doi.org/10.1002/adma.201605198

19. M. Rafiee, R.D. Farahani, D. Therriault, Multi-material 3D and 4D printing: a survey. Adv. Sci. 7(12), 1902307 (2020). https://doi.org/10.1002/advs.201902307

20. V.G. Rocha, E. Saiz, I.S. Tirichenko, E. García-Tuñón, Direct ink writing advances in multi-material structures for a sustainable future. J. Mater. Chem. A 8(31), 15646-15657 (2020). https://doi.org/10.1039/d0ta04181e

21. C.J. Zhang, L. McKeon, M.P. Kremer, S.H. Park, O. Ronan et al., Additive-free MXene inks and direct printing of microsupercapacitors. Nat. Commun. 10(1), 1795 (2019). https:// doi.org/10.1038/s41467-019-09398-1

22. W. Yang, J. Yang, J.J. Byun, F.P. Moissinac, J. Xu et al., 3D Printing of freestanding mxene architectures for currentcollector-free supercapacitors. Adv. Mater. 31(37), 1902725 (2019). https://doi.org/10.1002/adma.201902725

23. J. Orangi, F. Hamade, V.A. Davis, M. Beidaghi, 3D printing of additive-free $2 \mathrm{D} \mathrm{Ti}_{3} \mathrm{C}_{2} \mathrm{~T}_{\mathrm{x}}$ (MXene) ink for fabrication of micro-supercapacitors with ultra-high energy densities. ACS Nano 14(1), 640-650 (2020). https://doi.org/10.1021/acsnano. 9b07325

24. Z. Fan, C. Wei, L. Yu, Z. Xia, J. Cai et al., 3D printing of porous nitrogen-doped $\mathrm{Ti}_{3} \mathrm{C}_{2}$ MXene scaffolds for high-performance sodium-ion hybrid capacitors. ACS Nano 14(1), 867-876 (2020). https://doi.org/10.1021/acsnano.9b08030

25. X. Li, H. Li, X. Fan, X. Shi, J. Liang, 3D-printed stretchable micro-supercapacitor with remarkable areal performance. Adv. Energy. Mater. 10(14), 1903794 (2020). https://doi.org/ 10.1002/aenm.201903794

26. J.A. Lewis, Direct ink writing of 3D functional materials. Adv. Funct. Mater. 16(17), 2193-2204 (2006). https://doi.org/10. 1002/adfm.200600434

27. T.S. Wei, B.Y. Ahn, J. Grotto, J.A. Lewis, 3D printing of customized Li-ion batteries with thick electrodes. Adv. Mater. 30(16), 1703027 (2018). https://doi.org/10.1002/adma.20170 3027

28. T. Chen, W. Tang, J. Mu, T.J. Cui, Microwave metamaterials. Ann. Phys. 531(8), 1800445 (2019). https://doi.org/10.1002/ andp. 201800445

29. F. Calignano, D. Manfredi, E.P. Ambrosio, S. Biamino, M. Lombardi et al., Overview on additive manufacturing technologies. Proc. IEEE Inst. Electr. Electron. Eng. 105(4), 593-612 (2017). https://doi.org/10.1109/jproc.2016.2625098

30. Z. Wang, J. Ren, R. Liu, X. Sun, D. Huang et al., Three dimensional core-shell structured liquid metal/elastomer composite via coaxial direct ink writing for electromagnetic interference shielding. Compos. Part A: Appl. S. 136, 105957 (2020). https://doi.org/10.1016/j.compositesa.2020.105957

31. R.A. Shelby, D.R. Smith, S. Schultz, Experimental verification of a negative index of refraction. Science 292(6), 77-79 (2001). https://doi.org/10.1126/science.1058847

32. S.A. Nauroze, L.S. Novelino, M.M. Tentzeris, G.H. Paulino, Continuous-range tunable multilayer frequency-selective surfaces using origami and inkjet printing. Proc. Natl. Acad. Sci.
USA 115(52), 13210-13215 (2018). https://doi.org/10.1073/ pnas. 1812486115

33. B. Shen, Y. Li, D. Yi, W. Zhai, X. Wei et al., Strong flexible polymer/graphene composite films with 3D saw-tooth folding for enhanced and tunable electromagnetic shielding. Carbon 113, 55-62 (2017). https://doi.org/10.1016/j.carbon.2016.11. 034

34. M. Peng, Z. Wen, L. Xie, J. Cheng, Z. Jia et al., 3D printing of ultralight biomimetic hierarchical graphene materials with exceptional stiffness and resilience. Adv. Mater. 31(35), 1902930 (2019). https://doi.org/10.1002/adma.201902930

35. A. Lipatov, M. Alhabeb, M.R. Lukatskaya, A. Boson, Y. Gogotsi et al., Effect of synthesis on quality, electronic properties and environmental stability of individual monolayer $\mathrm{Ti}_{3} \mathrm{C}_{2} \mathrm{MXene}$ flakes. Adv. Electron. Mater. 2(12), 1600255 (2016). https://doi.org/10.1002/aelm.201600255

36. B. Akuzum, K. Maleski, B. Anasori, P. Lelyukh, N.J. Alvarez et al., Rheological characteristics of 2D titanium carbide (MXene) dispersions: a guide for processing MXenes. ACS Nano 12(3), 2685-2694 (2018). https://doi.org/10.1021/acsna no.7b08889

37. Y.Z. Zhang, Y. Wang, Q. Jiang, J.K. El-Demellawi, H. Kim et al., MXene printing and patterned coating for device applications. Adv. Mater. 32(21), 1908486 (2020). https://doi.org/ 10.1002/adma.201908486

38. H. Li, X. Li, J. Liang, Y. Chen, Hydrous $\mathrm{RuO}_{2}$-decorated MXene coordinating with silver nanowire inks enabling fully printed micro-supercapacitors with extraordinary volumetric performance. Adv. Energy Mater. 9(15), 1803987 (2019). https://doi.org/10.1002/aenm.201803987

39. M. Vural, A. Pena-Francesch, J. Bars-Pomes, H. Jung, H. Gudapati et al., Inkjet printing of self-assembled 2D titanium carbide and protein electrodes for stimuli-responsive electromagnetic shielding. Adv. Funct. Mater. 28(32), 1801972 (2018). https://doi.org/10.1002/adfm.201801972

40. L.H. Yu, Z.D. Fan, Y.L. Shao, Z.N. Tian, J.Y. Sun et al., Versatile N-doped MXene ink for printed electrochemical energy storage application. Adv. Energy. Mater. 9(34), 1901839 (2019). https://doi.org/10.1002/aenm.201901839

41. L. Li, Q. Lin, M. Tang, A.J.E. Duncan, C. Ke, Advanced polymer designs for direct-ink-write 3D printing. Chem. Eur. J. 25(46), 10768-10781 (2019). https://doi.org/10.1002/chem. 201900975

42. C. Zhu, T.Y. Han, E.B. Duoss, A.M. Golobic, J.D. Kuntz et al., Highly compressible 3D periodic graphene aerogel microlattices. Nat. Commun. 6, 6962 (2015). https://doi.org/10.1038/ ncomms7962

43. C. Zhu, J.E. Smay, Thixotropic rheology of concentrated alumina colloidal gels for solid freeform fabrication. J. Rheol. 55(3), 655-672 (2011). https://doi.org/10.1122/1.3573828

44. S.A. Shah, T. Habib, H. Gao, P. Gao, W. Sun et al., Templatefree $3 \mathrm{D}$ titanium carbide $\left(\mathrm{Ti}_{3} \mathrm{C}_{2} \mathrm{~T}_{\mathrm{x}}\right)$ MXene particles crumpled by capillary forces. Chem. Commun. 53(2), 400-403 (2017). https://doi.org/10.1039/c6cc07733a

45. V. Natu, M. Sokol, L. Verger, M.W. Barsoum, Effect of edge charges on stability and aggregation of $\mathrm{Ti}_{3} \mathrm{C}_{2} \mathrm{~T}_{\mathrm{z}}$ MXene 
colloidal suspensions. J. Phys. Chem. C 122(48), 2774527753 (2018). https://doi.org/10.1021/acs.jpcc.8b08860

46. Y. Deng, T. Shang, Z. Wu, Y. Tao, C. Luo et al., Fast gelation of $\mathrm{Ti}_{3} \mathrm{C}_{2} \mathrm{~T}_{\mathrm{x}}$ MXene initiated by metal ions. Adv. Mater. 31(43), 1902432 (2019). https://doi.org/10.1002/adma.201902432H

47. H. Chen, P. Ma, Y. Zhang, L. Wen Qu et al., Pristine titanium carbide mxene hydrogel matrix. ACS Nano 14(8), 1047111047 (2020). https://doi.org/10.1021/acsnano.0c04379

48. M. Ghidiu, J. Halim, S. Kota, D. Bish, Y. Gogotsi et al., Ionexchange and cation solvation reactions in $\mathrm{Ti}_{3} \mathrm{C}_{2}$ MXene. Chem. Mater. 28(10), 3507-3514 (2016). https://doi.org/10. 1021/acs.chemmater.6b01275

49. Z. Liu, Y. Zhang, H.B. Zhang, Y. Dai, J. Liu et al., Electrically conductive aluminum ion-reinforced MXene films for efficient electromagnetic interference shielding. J. Mater. Chem. C 8(5), 1673-1678 (2020). https://doi.org/10.1039/c9tc06304h

50. J. Liu, H.B. Zhang, R. Sun, Y. Liu, Z. Liu et al., Hydrophobic, flexible, and lightweight mxene foams for high-performance electromagnetic-interference shielding. Adv. Mater. 29(38), 1702367 (2017). https://doi.org/10.1002/adma.201702367

51. L. Lyu, J. Liu, H. Liu, C. Liu, Y. Lu et al., An overview of electrically conductive polymer nanocomposites toward electromagnetic interference shielding. Eng. Sci. 2, 26-42 (2018). https://doi.org/10.30919/es8d615

52. Z. Chen, D. Yi, B. Shen, L. Zhang, X. Ma et al., Semi-transparent biomass-derived macroscopic carbon grids for efficient and tunable electromagnetic shielding. Carbon 139, 271-278 (2018). https://doi.org/10.1016/j.carbon.2018.06.070S

53. S. Zhao, H.B. Zhang, J.Q.Luo, Q.W. Wang, B. Xu et al. Highly electrically conductive three-dimensional graphene oxide hybrid aerogels with excellent electromagnetic interference shielding performances. ACS Nano 12(11), 11193-11202 (2018). https://doi.org/10.1021/acsnano.8b05739

54. N. Wu, Q. Hu, R. Wei, X. Mai, N. Naik et al., Review on the electromagnetic interference shielding properties of carbon based materials and their novel composites: recent progress, challenges and prospects. Carbon 176, 88-105 (2021). https:// doi.org/10.1016/j.carbon.2021.01.124
55. X.X. Wang, W.Q. Cao, M.S. Cao, J. Yuan, Assembling nanomicroarchitecture for electromagnetic absorbers and smart devices. Adv. Mater. 32(36), 2002112 (2020). https://doi.org/ 10.1002/adma.202002112

56. B. Wen, M.S. Cao, Z.L. Hou, W.L. Song, L. Zhang et al., Temperature dependent microwave attenuation behavior for carbon-nanotube/silica composites. Carbon 65, 124-139 (2013). https://doi.org/10.1016/j.carbon.2013.07.110D

57. D.H. Park, J. Hong, I.S. Park, C.W. Lee, J.M. Kim, A colorimetric hydrocarbon sensor employing a swelling-induced mechanochromic polydiacetylene. Adv. Funct. Mater. 24(33), 5186-5193 (2014). https://doi.org/10.1002/adfm.201400779

58. J. Peng, Y. Cheng, A.P. Tomsia, L. Jiang, Q. Cheng, Thermochromic artificial nacre based on montmorillonite. ACS Appl. Mater. Interfaces 9(29), 24993-24998 (2017). https://doi.org/ 10.1021/acsami.7b07953

59. T. Habib, N. Patil, X. Zhao, E. Prehn, M. Anas et al., Heating of $\mathrm{Ti}_{3} \mathrm{C}_{2} \mathrm{~T}_{\mathrm{x}}$ MXene/polymer composites in response to radio frequency fields. Sci. Rep. 9(1), 16489 (2019). https://doi.org/ 10.1038/s41598-019-52972-2

60. P. He, M.S. Cao, Y.Z. Cai, J.C. Shu, W.Q. Cao et al., Selfassembling flexible 2D carbide MXene film with tunable integrated electron migration and group relaxation toward energy storage and green EMI shielding. Carbon 157, 80-89 (2020). https://doi.org/10.1016/j.carbon.2019.10.009

61. M. Cao, X. Wang, W. Cao, X. Fang, B. Wen et al., Thermally driven transport and relaxation switching self-powered electromagnetic energy conversion. Small 14(29), 1800987 (2018). https://doi.org/10.1002/smll.201800987S

62. S. Zhao, H. Wang, An integrated H-type method to measure thermoelectric properties of two-dimensional materials. ES Energy Environ. 9, 59-66 (2020). https://doi.org/10.30919/ esee8c262Z

63. Z. Ma, S. Kang, J. Ma, L.Shao, Y. Zhang et al., Ultraflexible and mechanically strong double-layered aramid nanofiber- $\mathrm{Ti}_{3} \mathrm{C}_{2} \mathrm{~T}_{\mathrm{x}}$ $\mathrm{MXene/silver} \mathrm{nanowire} \mathrm{nanocomposite} \mathrm{papers} \mathrm{for} \mathrm{high-perfor-}$ mance electromagnetic interference shielding. ACS Nano 14(7), 8368-8382 (2020). https://doi.org/10.1021/acsnano.0c02401 